\title{
CONTRIBUCIÓN AL ESTUDIO DE LA UTILIZACIÓN SELECTIVA POR APIS MELLIFERA L. DE LA FLORA LOCAL EN UN COLMENAR DEL NW DE LA PENÍNSULA IBÉRICA (GALICIA)
}

\author{
Emilia DIAZ LOSADA, Amelia V. GONZÁLEZ PORTO, Esperanza FERNÁNDEZ GÓMEZ \\ y Ma Pilar SAA OTERO
}

RESUMEN. Contribución al estudio de la utilización selectiva por Apis mellifera L. de la flora local en un colmenar del $N W$ de la Península Ibérica (Galicia). En el presente trabajo se ha estudiado la apetencia selectiva que Apis mellifera hace de las especies de la vegetación mediterránea subhúmeda de tendencia centroeuropea (según los índices climáticos de Alluè) en Galicia (NW de España), en su recolección de polen, mediante el muestreo sistemático de cúmulos de polen corbicular. Ello ha permitido diagnosticar y cuantificar las especies preferidas y determinar la importancia relativa de cada una de ellas.

Palabras clave. Polen apícola, melisopalinología, Galicia.

ABSTRACT. Contribution at the study of the selective utilization for Apis mellifera L. of the local flora in one apyary in the NW of Spain. In this work, we have determined the selective utilization of the pollen collection by Apis mellifera in the species of the sub-humed mediterranean (in accordance with climatic index of Alluè) zone of Galicia (NW of Spain), throughout sampling pollen loud at the hives. This was quantified the different plant used by honey bees, analyzing the relative importance of them.

Key words. Corbicular pollen, melysopalynology, Galicia.

\section{INTRODUCCIÓN}

Actualmente España es un país exportador de polen apícola, procedente principalmente de Extremadura y Castilla la Mancha. Dado el gran interés y desarrollo que está tomando la apicultura gallega y teniendo en cuenta que la mayor parte de las comarcas gallegas son aptas para la obtención de este tipo de producto, (Saa y Diaz, 1992), se han iniciado una serie de estudios sobre el tema, en los que se enmarca esta publicación.
Es bien sabido que las abejas son altamente selectivas y, fieles en su pecoreo, utilizan como fuente de polen parte de las plantas en flor disponibles (Free,1963), siendo éste su aporte primordial de proteínas y minerales y, mezclado con néctar y secreciones glandulares, la base de la alimentación de sus larvas (Von-Frisch, 1984; Sintes, 1987).

El objetivo de este trabajo es contribuir al conocimiento de las especies vegetales del territorio, mayoritariamente utilizadas por la abeja y, consecuentemente, a la determinación del origen floral del polen apícola. 


\section{MATERIAL Y MÉTODOS}

El estudio ha sido llevado a cabo en dos colmenas en Los Milagros (Orense-España), situadas a unos $200 \mathrm{~m}$ de distancia entre sí, pero con emplazamientos topográficos diferentes. Así la colmena 1 está situada en el borde de un pinar de Pinus pinaster Aiton, y la colmena 2 en un huerto, con cultivo de Brassica oleracea $\mathrm{L}$. fundamentalmente, existiendo frente e este colmenar una amplia zona con Genista tridentata L. y Erica umbellata L.

Se observó la evolución de la vegetación existente en el entorno de las colmenas en el período primaveral, según el método de cálculo de frecuencias de aparición de especies por unidad de superficie de muestreo y expresado en porcentaje relativo (Mueller \& Ellenberg, 1974). Para ello se trazaron 5 transectos lineales, de aproximadamente $300-1000 \mathrm{~m}$ de largo, abarcando las áreas más representativas de la vegetación, realizándose en ellos el seguimiento fenológico de especies (tab. 1) que, en principio, según estudios realizados anteriormente (Pérez et al., 1987; Battaglini \& Ricciardelli, 1970; Intoppa, et al., 1976-1977; Serra, 1988; Gomez, 1984; Hidalgo, et al., 1990) se espera sean utilizadas en la recolección de polen por la abeja. Para la elaboracion de la tabla fenológica se han seguido los criterios propuestos por Rachcke (1988).

El resultado de los transectos ha permitido cuantificar las especies mas abundantes en cada una de las formaciones vegetales presentes en el área de estudio: a) un pinar con Genista tridentata L., Erica umbellata L., Halimium alyssoides (Lam.) C. Koch, Calluna vulgaris (L.) Hull, Simethis planifolia (L.) Gren. y Ulex europaeus L.; b) un área higroturbosa con abundancia de Erica tetralix L., Calluna vulgaris (L.) Hull, Ulex minor Roth, Genista micrantha Ortega, Potentilla reptans L. y Senecio vulgaris L.; c) una amplia pradera con Tifolium repens L., Plantago lanceolata L., Mentha suaveolens Ehrh., Ranunculus repens
L. y Rumex acetosella L. y d) un matorral formado por Cytisus scoparius (L.) Link, Genista micrantha Ortega, Calluna vulgaris (L.) Hull, Cytisus multiflorus (L'Hér.) Sweet, Halimium alyssoides (Lam.) C. Koch, Potentilla reptans L., Erica tetralix L., Ulex minor Roth, Erica umbellata L., Simethis planifolia (L.) Gren., Halimium alyssoides (Lam.) C. Koch y Genista tridentata L.

Para la realización del estudio se tomaron muestras de cargas de polen corbicular, durante los meses de abril a septiembre y principios de octubre, mediante la instalación de trampas cazapolen en ambas colmenas, que se activaron durante un día, dos veces por mes, siempre que fue posible.

De cada recogida se tomó $1 \mathrm{~g}$ de peso y se sometió a acetolisis (Louveaux, et al., 1978.), con el fin de confeccionar preparaciones microscópicas, destinadas a obtener porcentajes de tipos polínicos identificados.

Se realizó separación de polen corbicular en base a su color, cotejados con una guia universal de colores PANTONE $747 \times \mathrm{R}$ (Hidalgo y Bootello, 1990). La monoespecificidad de cada cúmulo polínico se comprobó microscópicamente, tomando de 25 a 100 cúmulos, elegidos al azar, de cada uno de los conjuntos polínicos de coloración diferente. Se pesó cada uno de los conjuntos polínicos obtenidos para cada taxón y se hizo un cálculo porcentual de la biomasa que representa en el total de la recogida diaria.

\section{RESULTADOS Y DISCUSIÓN}

Los resultados del análisis de las cargas polínicas (tabs. 2 a 5) se representan de dos formas distintas: porcentajes relativos de biomasa para cada taxón identificado y porcentaje relativo de polen de taxones identificados en las preparaciones microscópicas de la muestra acetolítica. Estos datos representan los primeros aportados sobre 
ABRIL MAYO JUNIO JULIO AGOSTO SEPTIEMBRE OCTUBRE

Abies alba

Acer pseudoplatanus

Aesculus hipnocastanum

Anthemis arvensis

Aiuga pvramidalis

Brassica napus

Calluna vulgaris

Castanea sativa

Crataegus monogvna

Pvrus comunis

Cvtisus sconarius

Cvtisus multiflorus

Adenocarbus complicatus

Genista micrantha

Genista tridentata

Ulex europaeus

Ulex minor

Crenis canillaris

Taraxacum officinale

Echium vulgare

Echium lusitanicum

Erica arborea

Erica australis

Erica cinerea

Erica tetralix

Erica umbellata

Erodium cicutarium

Eucalvntus globulus

Eunhorbia heliosconia

Frangula alnus

Geranium molle

Halimium alvssoides

Hvpericum nerforatum

Jasione montana

Lactuca serriola

Lamium amplexicaule

Laurus nobilis

Ligustrum vulgare

Linum usitatissimum

Mentha suaveolens

Narcisus bulbocodium

Narcisus triandrus

Plantago coronodus

Plantago lanceolata

Prunus spinosa

Ouercus robur

Ouercus vvrenaica

Raphanus raphanistrum

Ranunculus repens

Reseda media

Rubus ulmifolius

Potentilla reptans

Salix fragilis

Sambucus nigra

Sedum acre

Senecio vulgaris

Scrophularia scorodonia

Scorzonera humilis

Simethis planifolia

Spergularia rubra

Stellaria media

Trifolium repens

Zea mavs
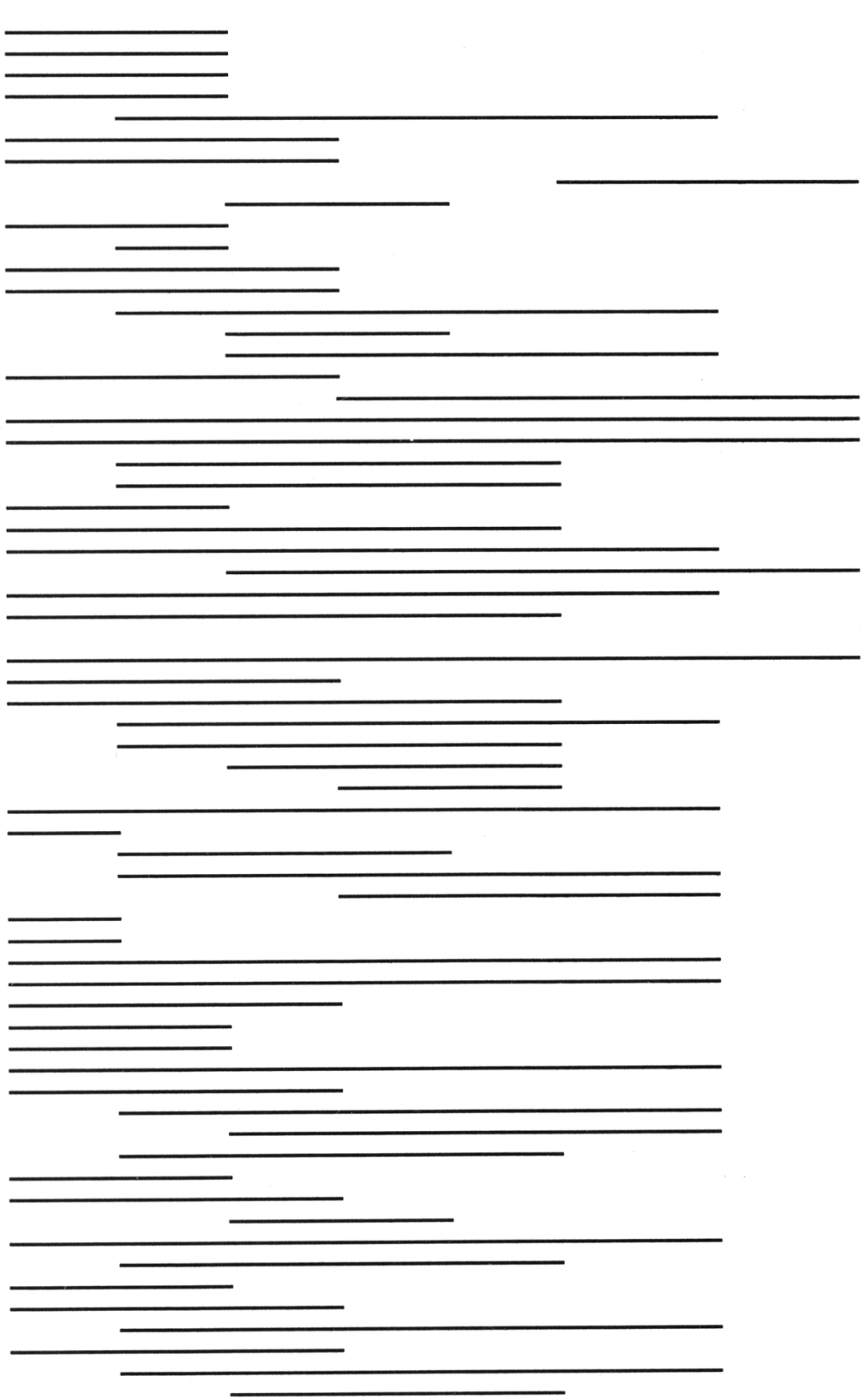

Tabla 1. Fenología de especies poliníferas, localizadas en el entorno de los colmenares. Phenologie of beeplant, locate in the proximity of the apyary. 


\begin{tabular}{|c|c|c|c|c|c|c|c|c|c|c|c|}
\hline TAXONES & & & & & RECOC & IDAS & & & & & \\
\hline & 1-IV & 2-IV & $1-V$ & 1-IV & 2-VI & $1-\mathrm{VII}$ & 2-VII & 1-VIII & 1-IX & 2-IX & $1-X$ \\
\hline Aesculus hippocastanum & & & 0,45 & & & & & & & & \\
\hline Acer pseudoplatanus & & 20,69 & & & & & & & & & \\
\hline Anthemis arvensis & & & & & 0,26 & & & & & & \\
\hline Brassica napus & 6,79 & & & & & & & & & & \\
\hline Calluna vulgaris & & & & & & & & & 61,78 & 64,03 & \\
\hline Castanea sativa & & & & & & 18,67 & & & & & \\
\hline Crataegus monogyna & & 21,21 & & & & & & & & & \\
\hline T. Cytisus scoparius & 78,65 & 29,89 & 48,10 & 28,19 & 0,85 & & 0,15 & 67,42 & 38,68 & 30,20 & 88,71 \\
\hline T. Echium vulgare & & & & & 1,74 & & & & & & \\
\hline Erica arborea & & & 1,12 & & & & & & & & \\
\hline Erica australis & & & & & & & & 3,47 & & & \\
\hline T. Erica cinerea & 7,14 & & & & & & & & & & \\
\hline Erica umbellata & & 17,12 & 0,63 & 69,25 & 59,69 & 31,71 & 1,18 & & & & \\
\hline Erodium cicutarium & & & & & 1,98 & & & & & & \\
\hline Eucalyptus globulus & 0,20 & & & 0,78 & 0,89 & 6,31 & & 15,00 & & 0,09 & 0,27 \\
\hline Frangula alnus & & & & 0,16 & & & & & & & \\
\hline Halimium alyssoides & & & 0,12 & 1,42 & 28,20 & & & & & & \\
\hline Jasione montana & & & & & 2,35 & & & & & & \\
\hline Lamium amplexicaule & 0,03 & & & & & & & & & & \\
\hline Laurus nobilis & & 1,53 & & & & & & & & & \\
\hline Linum usitatissimum & & & & & 2,27 & & & 15,08 & & & \\
\hline Ligustrum vulgare & & & & & 0,13 & & & & & & \\
\hline Mentha suaveolens & 5,24 & & & & & & & & & & \\
\hline Plantago lanceolata & & & & & 0,60 & & 8,47 & & & & \\
\hline Prunus spinosa & 0,08 & 0,30 & & & & & & & & & \\
\hline T. Quercus robur & & 10,23 & 15,32 & & & & & & & & \\
\hline Ranunculus repens & & 1,28 & 0,26 & & & & & & & & \\
\hline Raphanus raphanistrum & & 6,69 & & & & & & & & & \\
\hline Rubus ulmifolius & & & & & 1,04 & 23,65 & 10,08 & & & & \\
\hline Salix fragilis & 1,79 & & & & & & & & & & \\
\hline Sambucus nigra & & & 0,97 & & & & & & & & \\
\hline Spergularia rubra & & & 0,02 & 0,20 & & & & & & & \\
\hline Stellaria media & 3,10 & & & & & & & & & & \\
\hline Zea mays & & & & & & & 68,9 & & & & \\
\hline
\end{tabular}

Tabla 2. Porcentajes de biomasa para cada uno de los taxones identificados en las recogidas de la Colmena 1. El número arábigo indica primera o segunda recogida mensual respectivamente, el número romano el mes correspondiente. Biomass porcentage for each one of the taxon identified in the sample of the hive 1. The arabic numeral indicate first o second sample monthy, the roman numeral the month.

polen apícola procedente de colmenares gallegos.

Si bien ambos colmenares no pueden ser comparados en su totalidad, puesto que hubo de suspenderse el muestreo en la segunda colmena por causas fortuitas, si se pueden relacionar en el periodo común.

Los resultados muestran que las abejas han utilizado un total de 48 tipos polínicos diferentes en la primera colmena y 41 en la segunda, a lo largo de todo el periodo apícola, siendo diez las especies o tipos polínicos recogidos en mayor cantidad (porcentajes superiores al $10 \%$ ).

En ambas colmenas se observa que las abejas han ejercido una acción selectiva cualitativa y cuantitativa en la flora disponible. Así, el polen de T. Cytisus scoparius es el 


\begin{tabular}{|c|c|c|c|c|c|c|c|c|c|c|}
\hline \multirow[t]{2}{*}{ TAXONES } & \multicolumn{10}{|c|}{ RECOGIDAS } \\
\hline & $1-I V$ & $2-I V$ & $1-V$ & $1-V I$ & $2-\mathrm{VI}$ & $1-V I I$ & $2-V I I$ & 1-VIII & $1-I X$ & $2-I X$ \\
\hline Acer pseudoplatanus & & 6,32 & 1,44 & & & & & & & \\
\hline Aesculus hippocastanum & & 1,41 & 0,24 & & & & & & & \\
\hline $\begin{array}{l}\text { Anthemis arvensis } \\
\text { Ajuga pyramidalis }\end{array}$ & & & & & 0,31 & & & & & \\
\hline $\begin{array}{l}\text { Ajuga pyramidalis } \\
\text { Brassica napus }\end{array}$ & $\begin{array}{l}0,08 \\
7,32\end{array}$ & & & & & & & & & \\
\hline Calluna vulgaris & & & & & 1,51 & & & & 28,17 & 27,00 \\
\hline Castanea sativa & & & & & & 51,34 & 0,67 & 0,07 & & \\
\hline $\begin{array}{l}\text { T. Cytisus scoparius } \\
\text { T. Echium vulgare }\end{array}$ & 82,30 & 57,07 & 56,92 & 46,25 & $\begin{array}{c}1,43 \\
20,47\end{array}$ & & 3,95 & 90,54 & 71,58 & 73,00 \\
\hline $\begin{array}{l}\text { Erica arborea } \\
\text { T. Erica cinerea }\end{array}$ & & & & 2,54 & & & & & & \\
\hline $\begin{array}{l}\text { Erica umbellata } \\
\text { Eucalyptus globulus }\end{array}$ & 1,65 & 5,15 & 14,09 & 48,00 & 42,30 & 6,84 & $\begin{array}{l}0,59 \\
6,40\end{array}$ & 0,15 & & \\
\hline $\begin{array}{l}\text { Eucalyptus globulus } \\
\text { Frangula alnus }\end{array}$ & & & & 1,51 & 3,90 & 27,13 & & 8,73 & 0,07 & \\
\hline & & & & & 0,15 & & & & & \\
\hline $\begin{array}{l}\text { Geranium molle } \\
\text { Halimium alyssoides }\end{array}$ & & & & 0,15 & $\begin{array}{c}1,35 \\
20,31\end{array}$ & & & & & \\
\hline $\begin{array}{l}\text { Hypericum perforatum } \\
\text { Jasione montana }\end{array}$ & & 0,16 & & & & & & & & \\
\hline & & 0,08 & & 0,15 & 3,82 & & & & & \\
\hline $\begin{array}{l}\text { Lamium amplexicaule } \\
\text { Narcisus triandrus }\end{array}$ & 0,17 & $\begin{array}{l}0,24 \\
0,83\end{array}$ & & & & & & 0,08 & & \\
\hline $\begin{array}{l}\text { Narcisus triandrus } \\
\text { Poaceae }\end{array}$ & & 0,83 & & & & & & & & \\
\hline $\begin{array}{l}\text { Plantago coronopus } \\
\text { Plantago lanceolata }\end{array}$ & & & & & & & $\begin{array}{l}0,08 \\
2,95\end{array}$ & & & \\
\hline $\begin{array}{l}\text { Plantago lanceolata } \\
\text { Prunus spinosa }\end{array}$ & & & & & & & 28,58 & & & \\
\hline & 0,26 & 0,66 & 2,65 & & & & & & & \\
\hline $\begin{array}{l}\text { T. Quercus robur } \\
\text { Ranunculus repens }\end{array}$ & & $\begin{array}{l}2,16 \\
2,57\end{array}$ & $\begin{array}{c}16,74 \\
0,08\end{array}$ & & & & & & & \\
\hline Raphanus raphanistrum & & $\begin{array}{l}2,57 \\
8,48\end{array}$ & 0,08 & & 0,07 & & & & & \\
\hline $\begin{array}{l}\text { Rubus ulmifolius } \\
\text { Salix fragilis }\end{array}$ & & & & 0,15 & 3,42 & 14,66 & 35,58 & & & \\
\hline $\begin{array}{l}\text { Salix fragilis } \\
\text { Sambucus nigra }\end{array}$ & 6,19 & & & & & & & 0,07 & 0,07 & \\
\hline $\begin{array}{l}\text { Sambucus nigra } \\
\text { Scrophularia scorodonia }\end{array}$ & & $\begin{array}{l}0,24 \\
0,24\end{array}$ & 0,56 & 0,39 & 0,15 & & & & & \\
\hline $\begin{array}{l}\text { Senecio vulgaris } \\
\text { Spergularia rubra }\end{array}$ & & 0.24 & & 0,47 & 0,07 & & & & & \\
\hline $\begin{array}{l}\text { Spergularia rubra } \\
\text { Stellaria media }\end{array}$ & & 0,74 & 1,77 & & & & 0,08 & & & \\
\hline $\begin{array}{l}\text { Stellaria media } \\
\text { Trifolium repens }\end{array}$ & 2,09 & 0,08 & & & & & & & & \\
\hline Zea mays & & 0,00 & & & & & 20,48 & & & \\
\hline INDETERMINADOS & 0,08 & 0,33 & 0,24 & 0,07 & 0,31 & 0,08 & 0,08 & & 0,07 & \\
\hline
\end{tabular}

Tabla 3. Porcentajes de polen para cada uno de los taxones identificados en las recogidas de la Colmena 1. El número arábigo indica primera o segunda recogida mensual respectivamente, el número romano el mes correspondiente. The pollen porcentage for each one of the taxon identified in the sample of the hive 1. The arabic numeral indicate first o second sample monthy, the roman numeral the month.

recogido en mayor cantidad. Ello favorecido por el hecho de que las especies integrantes del T. Cytisus scoparius (Cytisus scoparius (L.) Link., Cytisus multuflorus (L'Hér.) Sweet, Adenocarpus complicatus (L.) Gay, Genista micrantha Ortega, Genista tridentata L., Ulex europaeus L. y Ulex minor Roth), se suceden en sus floraciones desde abril a octubre y son muy abundantes en el entorno. Estas circunstancias, juntamente con la importancia de su valor nutritivo son, según Sintes (1987), las condiciones óptimas para que sean fuente preferida por la abeja. Hay excepciones como en los casos siguientes: para la colmena 1 la segunda recogida de junio en la que que son mayoritarios Erica umbellata L., T. Echium vulgare y Halimium alyssoides (Lam.) C. Koch; en la primera de julio, Castanea sativa Miller, 


\begin{tabular}{|c|c|c|c|c|c|c|c|}
\hline \multirow[t]{2}{*}{ TAXONES } & \multicolumn{7}{|c|}{ RECOGIDAS } \\
\hline & $1-I V$ & 2-IV & $1-\mathrm{V}$ & $1-\mathrm{VI}$ & $2-\mathrm{VI}$ & 1-VII & $1-$ VIII \\
\hline Castanea sativa & & & & & & 59 & \\
\hline T. Crataegus monogyna & 2,51 & & 5,06 & & & & \\
\hline T. Crepis capillaris & 1,16 & 13,24 & & & & & 1,79 \\
\hline T. Cytisus scoparius & 25,37 & 55,37 & 38,20 & 50,68 & & & 92,79 \\
\hline T. Echium vulgare & & & & 2,06 & 16,14 & 0,49 & \\
\hline Erica arborea & & & 0,70 & & & & \\
\hline T. Erica cinerea & & & & & & & 0,21 \\
\hline Erica umbellata & 20,00 & 9,57 & 24,07 & 23,93 & 18,94 & 2,00 & \\
\hline Eucalyptus globulus & & & 0,45 & 4,16 & 12,36 & 7,70 & \\
\hline Euphorbia helioscopia & & & & 0,98 & & & \\
\hline Halimium alyssoides & & & & 16,48 & 29,95 & 0,14 & \\
\hline Linum usitatissimun & & & & & & 0,59 & 0,12 \\
\hline Mentha suaveolens & 0,74 & & & & & & \\
\hline Narcisus bulbocodium & 0,028 & & & & & & \\
\hline Prunus spinosa & 6,96 & 0,67 & & & & & \\
\hline T. Quercus robur & & & 1,05 & 0,11 & & & \\
\hline Ranunculus repens & & & 9,37 & & & & \\
\hline Raphanus raphanistrum & 21,61 & 5,52 & & & & & \\
\hline Rubus ulmifolius & & & & & 1,95 & 22,07 & \\
\hline Salix fragilis & 1,88 & & & & & & \\
\hline Sedum acre & & & 0,09 & & & & \\
\hline Simethis planifolia & & & & 0,2 & & & \\
\hline Spergularia rubra & & & 4,8 & 0,06 & 4,45 & & \\
\hline Stellaria media & 0,73 & & & & & & \\
\hline Trifolium repens & & & & & & 13.25 & \\
\hline
\end{tabular}

Tabla 4. Porcentajes de biomasa para cada uno de los taxones identificados en las recogidas de la Colmena 2. El número arábigo indica primera o segunda recogida mensual respectivamente, el número romano, el mes correspondiente. Biomass porcentage for each one of the taxon identified in the sample of the hive 2. The arabic numeral indicate first o second sample monthy, the roman numeral the month.

Rubus ulmifolius Schott en y Eucalyptus globulus Labill.; y en la segunda de julio Zea mays L., y Rubus ulmifolius Schott; para la colmena 2 la segunda de junio en que son preferentes T. Echium vulgare, Erica umbellata L. Eucalyptus globulus Labill. y Halimium alyssoides (L.) C. Koch; en la primera de julio Castanea sativa Miller y Rubus ulmifolius Schott.

Se observan ligeras diferencias en el comportamiento selectivo de ambas colmenas en relación con sus apetencias de T. Raphanus raphanistrum, Eucalyptus globulus Labill., y Erica umbellata L.

Existen también diferencias cualitativas y cuantitativas en los porcentajes de biomasa y en los porcentajes de granos de polen en las preparaciones acetolíticas, atribuibles a la falta de correlación entre el peso de cargas y cantidad de polen presente en dichas cargas en función del tamaño de polen de cada especie (Louveaux, 1958-1959).

La cuantificación de especies presentes en el polen corbicular ha permitido determinar la frecuencia relativa de cada una de ellas. Mención aparte merece la predilección mostrada por Castanea sativa Miller cuyos porcentajes llegan a alcanzar $87.34 \%$; T. Cytisus scoparius, hasta $95.83 \%$; Erica umbellata L., $69.25 \%$; Zea mays L., $68.9 \%$; etc. 


\begin{tabular}{|c|c|c|c|c|c|c|}
\hline TAXONES & & & ECOGII & & & \\
\hline & 1-IV & 2-IV & $1-\mathrm{V}$ & $1-\mathrm{VI}$ & $1-$ VII & 1-VIII \\
\hline Acer psedoplatanus & 0,37 & & 6,27 & & & \\
\hline $\begin{array}{l}\text { Ajuga pyramidalis } \\
\text { Brassica napus }\end{array}$ & & 0,09 & & & & \\
\hline $\begin{array}{l}\text { Brassica napus } \\
\text { Calluna vulgaris }\end{array}$ & & 0,83 & & & & \\
\hline $\begin{array}{l}\text { Calluna vulgaris } \\
\text { Castanea sativa }\end{array}$ & & & & & 87,34 & 0,1 \\
\hline T. Crataegus monogyna & 0,09 & & & & & \\
\hline T. Crepis capillaris & 0,27 & 9,35 & & & & \\
\hline $\begin{array}{l}\text { T. Cytisus scoparius } \\
\text { T. Echium vulgare }\end{array}$ & 37,00 & 68,98 & 57,72 & $\begin{array}{l}61,35 \\
9,66\end{array}$ & & 95,63 \\
\hline Erica arborea & & & 0,60 & & & \\
\hline $\begin{array}{l}\text { Erica umbellata } \\
\text { Erodium cicutarium }\end{array}$ & 7,28 & $\begin{array}{l}1,20 \\
0,55\end{array}$ & 11,94 & 14,69 & & \\
\hline Eucalyptus globulus & & $\begin{array}{l}0,55 \\
0,09\end{array}$ & 2,31 & 7,43 & 1,29 & 0,10 \\
\hline $\begin{array}{l}\text { Halimium alyssoides } \\
\text { Hypericum perforatum }\end{array}$ & & 0,09 & 0,85 & 4,78 & & \\
\hline Lactuca serriola & 0,09 & & & & & \\
\hline Lamium amplexicaule & 0,09 & 0,09 & & & & \\
\hline $\begin{array}{l}\text { T. Orlaya daucoides } \\
\text { Plantago lanceolata }\end{array}$ & & 0,09 & & & 0,51 & 0,1 \\
\hline $\begin{array}{l}\text { Poaceae } \\
\text { Prunus spinosa }\end{array}$ & & & & & 0,17 & \\
\hline $\begin{array}{l}\text { Prunus spinosa } \\
\text { T. Quercus robur }\end{array}$ & 4,44 & 1,75 & 4,98 & & & \\
\hline $\begin{array}{l}\text { T. Quercus robur } \\
\text { Ranunculus repens }\end{array}$ & 0,18 & 0,09 & $\begin{array}{l}1,80 \\
0,08\end{array}$ & 0,24 & & \\
\hline $\begin{array}{l}\text { Raphanus raphanistrum } \\
\text { Reseda }\end{array}$ & 44,74 & 9,90 & $\begin{array}{l}0,08 \\
0,34\end{array}$ & 0,08 & & \\
\hline $\begin{array}{l}\text { Rubus ulmifolius } \\
\text { T. Rumex }\end{array}$ & & & & 0,57 & 8,26 & \\
\hline $\begin{array}{l}\text { T. Rumex } \\
\text { Salix fragilis }\end{array}$ & 4,54 & 0,25 & 0,08 & & & \\
\hline $\begin{array}{l}\text { Scorzonera humilis } \\
\text { Simethis planifolia }\end{array}$ & & 0,09 & & & & \\
\hline $\begin{array}{l}\text { Simethis planifolia } \\
\text { Spergularia rubra }\end{array}$ & & 4,72 & 2,23 & 0,08 & & \\
\hline $\begin{array}{l}\text { Stellaria media } \\
\text { Trifolium repens }\end{array}$ & 0,85 & & & & 2,49 & \\
\hline INDETERMINADOS & 0,09 & 0,64 & 0,34 & 0,74 & & 0,1 \\
\hline
\end{tabular}

Tabla 5. Porcentajes de polen para cada uno de los taxones identificados en las recogidas de la Colmena 2. El número arábigo indica primera o segunda recogida mensual respectivamente, el número romano el mes correspondiente. The pollen porcentage for each one of the taxon identified in the sample of the hive 2 . The arabic numeral indicate first o second sample monthy, the roman numeral the month.

\section{BIBLIOGRAFÍA}

BATTAGLINI, M. \& G. RICCIARDELLI -1970Sulla flora pollinifera di alcune zone dell'Umbria. Note e App. Spe. di Ent. Agr. 13:124.

FREE, J.B. -1963- The flower constancy of honey bee. J. Anim. Ecol. 32:119-131.

GÓMEZ-FERRERAS, C. -1984- Origen botánico del polen comercializado en España. II Congreso
Nac. de Apicultura. Gijon. 70-93.

HIDALGO, M.I., M.L. BOOTELLO y J. PACHECO -1990- Origen floral de las cargas de polen recogidas por Apis mellifera $\mathrm{L}$. en Alora (Malaga,España). Acta Botánica Malacitana. 15:33-34

HIDALGO, M.I. y M.L. BOOTELLO -1990- About some physical characteristics of the pollen loads collected by Apis mellifera L. Apicultura. 6:179191. 
INTOPPA, F., L. PERSSANO-ODDO \& G. RICCIARDELLI -1976-1977- Indagine sulla flora pollinifera delle regioni Marche e Toscana. Ann. Ist. Super. Zool. Agr. (5):33-44.

LOUVEAUX, J., A. MAURIZZIO \& G. VORDWOHL -1978-. Methods of melissopalynology. Bee World. 59(4):139-157.

LOUVEAUX, J. -1958-1959-. Recherches sur la récolte du pollen per les abeilles (Apis mellifera L.). Ann. Abeille. 1(3):113-188; 1(4):197-221; 2(1):13-111.

MUELLER-DOMBOIS, D. \& H. ELLENBERG 1974-Aims and methodos of vegetation ecology. Ed. John Wiley \& Sons. Nueva York.

PÉREZ-GARCÍA, F., A. GÓMEZ-PAJUELO y J.L. MOLINS-MARÍN -1987- Origen floral y coloración de las pelotas de polen. Vida Apícola. 25:33-45.

RATHCKE, B. -1988- Flowering phenologies in a shrub community: competition and constraints. Journal of Ecology. 76:975-994.

SAA-OTERO, M.P. y E. DÍAZ-LOSADA -1992Polen apícola: origen, propiedades y utilidades. I Congreso Apícola Gallego. Monforte. Galicia. SERRA-BONVEHI, J. -1988- Origen botánico del polen apícola producido en España. An. Asoc. Pal. Leng. Esp. 4:73-78.

SINTES-PROSS, J. -1987-. Las plantas melíferas y la alimentación de las abejas. Ed. Sintes. Barcelona.

VON-FRISCH, K. -1984- La vida de las abejas. Ed. Labor. Barcelona.

Aceptado para su publicación en Octubre de 1994

Dirección de las autoras. Facultad de Ciencias de Orense. Universidad de Vigo. 32004 Orense. 